\title{
An overview of the current three-dimensional body scanners for anthropometric data collection
}

\author{
S. Bragança \\ University of Minho, Guimarães, Portugal \\ P. Arezes \\ University of Minho, Guimarães, Portugal \\ M. Carvalho \\ University of Minho, Guimarães, Portugal
}

\begin{abstract}
Three-dimensional body scanners have revolutionized the anthropometric data collection. These data can be used for numerous applications, in different fields of expertise. However, there are different types of technologies and products to be used, influencing the results obtained. This paper presents a non-exhaustive synopsis of the existing technologies and products available in the market.
\end{abstract}

\section{INTRODUCTION}

With the diffusion of digital devices for body scanning, the science of anthropometry gained a new powerful tool that allows deeper investigations on the human body shape. The new digital shape analysis tools allow rethinking the anthropometry science since, in the digital environment, the measurements are not limited to the traditional one dimensional ones, and instead it is possible to have complex geometrical features (i.e. curvatures and partial volumes). 3D body scanners have revolutionized anthropometric data acquisition, being more practical, accurate, fast and, when compared to traditional anthropometry, less expensive.

The purpose of this paper is to present an overview of the currently existing body scanners, the available types of technology and the standard process of scanning test subjects.

\section{OVERVIEW}

The study of the human body as a 3D object has only started in 1973 with a light sectioning technique proposed by Lovesey (Lovesey, 1966). This was labor intensive as the interpretation of data was extremely time consuming. That technology evolved to what is now known as a three-dimensional body scanner.

A whole body scanner is an optical 3D measuring system that produces a digital copy of the surface geometry of the human body (Daanen and Water, 1998). In most cases, threedimensional body scanners capture the outside surface of the human body by using optical techniques, in combination with light sensitive devices, without physical contact with the body. The subject being scanned usually wears form-fitting clothes during the process. This means that there is no longer the need for physical contact with the subject's body. However, the image based data collection introduces the question of privacy. There are different opinions regarding the privacy of the body scanner. If in the one hand it provides more privacy, since it avoids the need to actually touch the body, on the other hand the highly accurate, more personal, images produced by the scanners are potentially more invasive since they can be stored insecurely and transferred directly from the scanner over local networks or the internet. Neverthe- 
less, with these advances in the anthropometric science and computer-based human-form modeling it is now possible to give a different perspective to the collection of anthropometric measurements.

One of the earliest 3D body scanning systems was a shadow scanning method developed by the Loughborough University in the UK, the Loughborough Anthropometric Shadow Scanner LASS - (Jones et al., 1989). This system was developed and used to digitize the human body, but it was necessary to manipulate the data in order to take body measurements from the scan. The original shadow scanning methods are different from other conventional structured lighting approaches since they require very little hardware besides a camera, a desk-lamp, a pencil and a checkerboard. LASS was an automated, computerized 3D measurement system based on triangulation, where the subjects stood on a rotating platform that was turned $360^{\circ}$ in measured angular increments. Brooke-Wavell et al. (1994) compared anthropometric measurements taken with LASS with measurements taken using traditional anthropometry and concluded that they were similar. For women, statistical differences were found between various measurements (neck and chest circumferences, waist width, depth and height), whilst for men a significant difference was only found between the measurements of waist depth. These differences were explained as being due to landmarking and to difficulties in making horizontal measurements with the tape measure.

\subsection{Types of imaging techniques}

Currently, there are several types of imaging techniques to create full body images. These imaging technologies, include (i) 2D video silhouette images converted to 3D models, (ii) white light phase based image capture, (iii) laser-based image capture, and (iv) radio-wave linear array image capture (Treleaven and Wells, 2007; Istook and Hwang, 2001). Body scanning systems normally consist of one or more light sources, one or more vision or capturing devices, software, computer systems and monitor screens to visualize the data capture process (Daanen and Water, 1998). There are several types of body scanning technologies but the major ones are those using laser and light. Table 1 briefly describes the various types of technology available for three-dimensional body scanners (Daanen and Haar, 2013).

Table 1 . Types of technology used by 3D body scanners.

\begin{tabular}{ll}
\hline Type of technology & Description \\
\hline Laser line systems & A laser line is projected on the body from different sides and viewed by \\
cameras under a fixed angle. The advantage of a single line is that the sen- \\
sor can easily detect it and very accurately compute how the projected 2D \\
line is deformed on the 3D surface. The sequential 3D lines then form the \\
complete 3D image.
\end{tabular}

Structured light systems

A structured light system projects a full structured light pattern into the scene and, from the sensed deformed pattern, a full 3D image is calculated. This pattern can consist of dots, bars, or any other light patterns. The advantage of a structured light scanner is its speed. Structured light scanning is so fast, that it can be used for 4D scanning; i.e. real time 3D scanning at $200 \mathrm{~Hz}$. This offers nice opportunities to couple movement registration to $3 \mathrm{D}$ shape analysis.

Multi-view camera system A $\quad$ AD image is acquired from two or more cameras. A stereo-camera records two images at the same time from a different viewpoint. From the content in the two images the depth to the body can be calculated and converted into a dense 3D image in real-time. The advantage of a stereocamera system is that no laser line or light pattern is transmitted, which means that sunlight cannot interfere with the pattern. However, using the line or patterns enables a 3D image with higher resolution and accuracy.

Millimeter waves There are active and passive millimeter wave scanners. Active scanners use the reflection patterns of millimeter waves projected on the body. Passive scanners process the millimeter waves that are emitted by the human skin. Millimeter waves offer the advantage that they pass through most clothing ensembles but not the skin. Thus, the shape of the body can be captured without undressing. This offers an advantage in time and effort, but may introduce an ethical problem because the private parts of the subjects can be seen. Millimeter wave scanners are currently employed at airports for the detection of metal parts under garments and offer an alternative for low radiation x-ray scanners. 
It is possible to find many studies that compared the various types of existing body scanners. Jones and Rioux (1997), Daanen and Water (1998), Olds and Honey (2005) and Daanen and Haar (2013) discuss the use of 3D whole body scanners in anthropometry as a whole, giving a good overview of the evolution of body scanning technology and the different scanners in use at the time. Olds and Honey (2005) affirm that scanners using white light are generally faster and cheaper than laser scanners, but can produce lower quality scans, with areas of data missing. Despite concluding that body scanners are expensive, require technical expertise, cannot measure skin-folds or compressed bone lengths, they agree that body scanners offer the ability to collect greater amounts of data, extract data when the subjects are no longer present and use the data directly in computer-aided design software applications. McKinnon and Istook (2001) compared two scanners available at that time from TC2, finding that the newer scanner was an improvement on the older scanner, producing data that replicated more closely the one obtained using traditional measurement methods. Then they anticipated that the extraction of fast and accurate anthropometric data would be possible in the future, as in fact it is nowadays.

\subsection{The different body scanners}

Based in a publication from 2013 from Daanen and Haar, there are currently available on the market various 3D whole body scanning systems, as the ones presented in Table 2.

Table 2. Currently available 3D whole body scanning systems (Daanen and Haar, 2013).

\begin{tabular}{llll}
\hline Company & Product & City, Country & \multicolumn{1}{c}{ Technique } \\
\hline Cyberware & WBX & Monterey, CA, USA & Laser line \\
4ddynamics & Mephisto EX-pro or CX-pro & Antwerp, Belgium & Structured light projection \\
4ddynamics & Gotcha & Antwerp, Belgium & Structured light projection \\
Vitronics & Vitrus Smart LC & Wiesbaden, Germany & Laser line \\
Vitronics & Vitrus Smart XXL & Wiesbaden, Germany & Laser line \\
TC2 & KX-16 & Cary, NC, USA & Infrared \\
SizeStream & 3D body scanner & Cary, NC, USA & Infrared \\
SpaceVision & Cartesia & Tokyo, Japan & Laser structured light \\
3dMDbody & Flex8 & Atalanta, GA, USA & Stereo photogrammetry \\
\hline
\end{tabular}

There are some new ways of creating three-dimensional images using systems that were not initially designed to do so. This is the case of Microsoft Kinect that can be used for numerous other applications besides games. The Microsoft Kinect sensor has its place in the class of devices known as depth cameras and fall under the structured light systems categorization regarding the type of technology used (Shotton et al., 2011). Kinect can be seen as a 3D marker-less motion capture system because it provides a simplified skeleton in real time without the need for especial clothes or other equipment. Despite the fact that it cannot be used for extremely accurate studies (Ye et al., 2011; Clark et al., 2012; Bragança et al., 2014), it can be used when there is no need for high accuracy, like in clothes or shoes sizing, indirect fat measurement or clinical rehabilitation (Baena et al., 2012; Weiss et al., 2011).

\subsection{Landmarking}

Before starting the scanning process it is necessary to identify some body parts to simplify the measuring process - this process is often called landmarking. Landmarking is, indeed, a subject very much discussed throughout the research.

For the purpose of landmarking and body dimensions' collection, the 3D human model is usually segmented at armpits and crotch (process known as segmentation). Five body parts including head and torso, both arms, and both legs can be identified. There are many ways to perform this segmentation, for example, Nurre et al. (2000) proposed a cusp algorithm for segmenting the 3D scanning data of a human body and Wang et al. (2007) applied fuzzy logic concept to locate the armholes and crotch, and then to separate the arms and legs from the trunk.

After segmentation, the scanning data will be ready for landmarking. Landmarking's objective is to find out the anatomical landmarks that are used to define body size and shape (Figure 1). Since landmarks are mostly bony protrusions, palpation works are needed for identification. 
In general, anthropometric data technicians place markers or stickers on the surface of human body to highlight the positions.

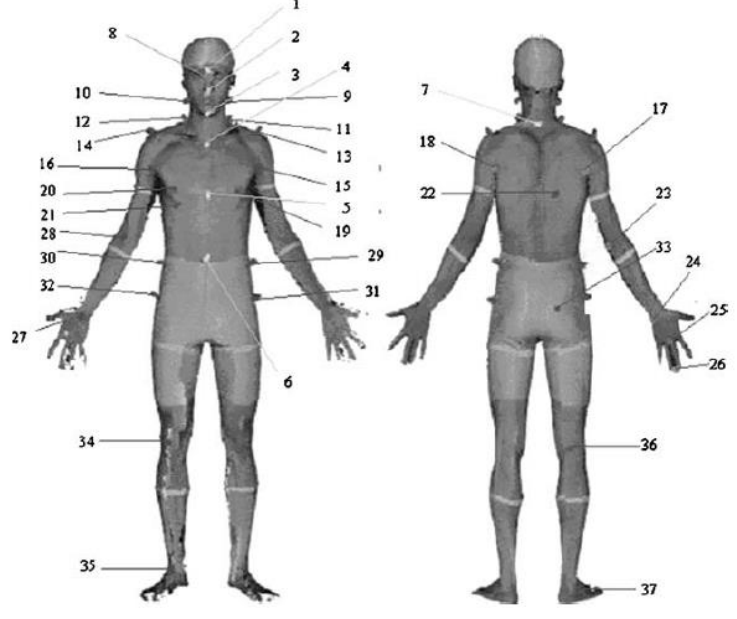

Figure 1. Landmarks used for collecting the anthropometric dimensions (Wang et al., 2007).

As this is a process that requires some training, some attention has been given to developing the methods to facilitate correct landmark identification. According to Wang et al. (2007), the landmark identification methods can be generally classified as: (i) premarking, (ii) human body mapping, (iii) geometry analysis and (iv) approximate height location determination. Thus the positions of the landmarks can be easily identified on the scanning image with human eyes. Further, by using the color information obtained from the cameras in the scanning heads, the landmarks can also be identified by analyzing the RGB information in the scanning image (Daanen and Water, 1998; Burnsides et al., 2001; Wang et al., 2007).

\subsection{Applications}

Body scanners are used for a wide variety of applications. Jones and Rioux (1997) divided their applications in:

- Medical: body deformity; glaucoma; orthodontics; orthopedics; surgery; lung function studies; custom prostheses; breast topography; pediatrics; medical management;

- Human systems engineering: work environment; population anthropology; helmets and face masks; gloves; clothing; human morphology; human motion analysis; forensic imaging; hearing studies;

- Virtual reality and communications: three-dimensional portraits; computer animation of human models.

According to Ashdown et al. (2004), more recently, 3D scans have been used to create virtual models of customers of the apparel industry, which consumers can manipulate to mirror their own bodies. Consumers can then try clothing on their model online

\section{CONCLUSIONS}

Creating anthropometric databases typically requires considerable resources (time, know-how, funds, equipment and workforce). To overcome these limitations the technological development in recent years, using three-dimensional forms, allowed the study of human size and shape to be done in a more expeditious and efficient way that requires fewer resources. 3D body scanners make the anthropometric data acquisition more practical, accurate, fast and less expensive. With these advances it is now possible to explore and give a different perspective to the use of anthropometric measurements.

3D scanning systems have evolved over the last few years. From LASS to Kinect the technology is always in progress. The type of technology used is different from scanner to scanner, existing four main types of technology - laser line systems, structured light systems, multi-view 
camera system and millimeter waves. Currently, there is a relatively high variety of threedimensional body scanners available in the market (nine major products developed all over the world).

According to the technology and product used, the results and applications are different. Different products may have different accuracy values so the applicability of the data may be compromised. Depending on the desired application, i.e., highly precise anthropometric data or less accurate data for apparel applications, there is a variety of body scanners that can be used. For example the Microsoft Kinect, that is less accurate, can be used for apparel applications, while the Vitrus Smart LC, that is more precise, can be used for compiling an anthropometric database.

\section{REFERENCES}

Ashdown, S. P., Loker, S., Schoenfelder, K. \& Lyman-Clarke, L. 2004. Using 3D scans for fit analysis. Journal of Textile and Apparel, Technology and Management, 4(1): 1-12.

Baena, A., Susín, A. \& Lligadas, X. (2012). Biomechanical validation of upper-body and lower-body joint movements of kinect motion capture data for rehabilitation treatments. 4th International Conference on Intelligent Networking and Collaborative Systems (INCoS), 656-661.

Bragança, S., Carvalho, M., Xu, B., Arezes, P \&Ashdown, S. 2014. A Validation Study of a Kinect Based Body Imaging (KBI) Device System Based on ISO 20685:2010, Proceedings of the fifth International Conference on 3D Body Scanning Technologies, Lugano, Switzerland.

Brooke-Wavell, K., Jones, P.R.M. \& West, G.M. 1994. Reliability and repeatability of 3-D body scanner (LASS) measurements compared to anthropometry. Annals of Human Biology. 21(6): 571-577.

Burnsides, D., Boehmer, M. \& Robinette, K. 2001. 3-D landmark detection and identification in the CAESAR project, Proceedings of the third international conference on 3-D digital imaging and modeling conference, Quebec City.

Clark, R. A., Pua, Y. H., Fortin, K., Ritchie, C., Webster, K. E., Denehy, L. \& Bryant, A. L. 2012. Validity of the Microsoft Kinect for assessment of postural control. Gait \& posture, 36(3):372-377.

Daanen, H. A. M. \& Ter Haar, F. B. 2013. 3D whole body scanners revisited. Displays, 34(4): 270-275.

Daanen, H. M. \& van de Water, G. J. 1998. Whole body scanners. Displays, 19(3):111-120.

Istook, C. L. \& Hwang, S. J. (2001). 3D body scanning systems with application to the apparel industry. Journal of Fashion Marketing and Management, 5(2):120-132.

Jones, P. R. \& Rioux, M. 1997. Three-dimensional surface anthropometry: applications to the human body. Optics and Lasers in Engineering, 28(2):89-117.

Jones, P.R.M., West, G.M., Harris, D.H. \& Read, J.B. 1989. The Loughborough anthropometric Shadow scanner (LASS). Endeavour 13: 162-168.

Lovesey, E. J., A method of determining facial contours by shadow projection. 1966. Royal Aircraft Establishment Technical Report, 66192.

Mckinnon, L. \& Istook, C. 2001. Comparitive analysis of the image twin system and the 3T6 body scanner. Journal of textile and apparel, Technology and Management, 1(2):1-7.

Nurre, J. H., Connor, J., Lewark, E. A. \& Collier, J. S. 2000. On segmenting the three-dimensional scan data of a human body. IEEE Transactions on Medical Imaging, 19(8):787-797

Olds, T. \& Honey, F. 2005. The use of 3D whole-body scanners in anthropometry, Kinanthropometry IX. Routledge, London and New York, 1-12.

Shotton, J., Sharp, T., Kipman, A., Fitzgibbon, A., Finocchio, M., Blake, A. \& Moore, R. 2013. Real-time human pose recognition in parts from single depth images. Communications of the ACM, 56(1):116124.

Treleaven, P. \& Wells, J. C. K. 2007. 3D body scanning and healthcare applications. Computer, 40(7):2834.

Wang, M. J. J., Wu, W. Y., Lin, K. C., Yang, S. N. \& Lu, J. M. 2007. Automated anthropometric data collection from three-dimensional digital human models. The International Journal of Advanced Manufacturing Technology, 32(1-2):109-115.

Weiss, A., Hirshberg, D. \& Black, M. J. (2011). Home 3D body scans from noisy image and range data. IEEE International Conference on Computer Vision (ICCV), 1951-1958).

Ye, M., Wang, X., Yang, R., Ren, L. \& Pollefeys, M. (2011). Accurate 3d pose estimation from a single depth image. IEEE International Conference on Computer Vision (ICCV), 731-738. 